\title{
DESIGN AND QUALITATIVE EVALUATION OF TACTILE DEVICES FOR STROKE REHABILITATION
}

\author{
G.V. Merrett**1 C.D. Metcalf* ${ }^{\dagger}$, D. Zheng*, S. Cunningham ${ }^{\dagger}$, S. Barrow*, S.H. Demain ${ }^{\dagger}$ \\ * Electronics and Computer Science, University of Southampton, Hampshire, UK, SO17 1BJ \\ ${ }^{\dagger}$ Faculty of Health Sciences, University of Southampton, Hampshire, UK, SO17 1BJ \\ ${ }^{1}$ E-mail: gvm@ecs.soton.ac.uk
}

Keywords: tactile, haptic, stroke, shape memory alloy

\begin{abstract}
Rehabilitation environments combining virtual reality with everyday motor tasks can promote recovery from neurological illness, such as stroke. Tactile devices, providing physical stimulation to the skin, may improve motor retraining. While many tactile devices have been reported, there is a distinct paucity of studies evaluating how they are perceived. This multidisciplinary research has investigated three tactile devices (vibration motors, a motor-driven 'squeezer', and shape memory alloys) for providing a realistic sensation of static interaction with virtual objects. These devices have been iteratively redesigned and qualitatively evaluated with healthy human participants. This paper presents the devices, their evaluation, and iterative redesign.
\end{abstract}

\section{Introduction}

Rehabilitation environments that combine virtual reality with everyday motor tasks can promote recovery from neurological illness, such as stroke [1]. Intact sensation is essential for a multitude of activities including fine motor control [2], injury prevention [3] and a normal quality of life. Haptic devices are used to provide artificial stimulation to the skin to generate a 'sense of touch', and may offer more realistic and immersive virtual environments, subsequently leading to improved motor retraining. Therefore, haptic devices are increasingly incorporated into therapy to retrain sensory abilities [4] to convey properties of virtual objects (enhancing the realism of the sensorial experience) [5].

Haptic sensations can be broadly classified as coming from two distinct inputs, namely kinaesthetic and cutaneous stimuli [6]. Kinaesthetic inputs are generated by stimulating receptors in the muscles, joints and tendons, and represent movement, position and posture. Cutaneous (or, as referred to in this paper, tactile) inputs are generated by stimulating mechanoreceptors in the skin, and detect skin contact with objects and perception of surface properties. Clearly, the perception of touching an object relies on both of these inputs; consider picking up a box, the skin on the finger and hand is stimulated by the deformation of the fingertip (tactile), and the finger joints, muscles and tendons detect the normal force exerted through gripping (kinaesthetic). However, in this research we are concerned only with generating realistic tactile sensations, as this does not require intrusive hardware such as mechanical exoskeletons [7]. Whilst the ultimate aim of this research is an integrated sensory and motor rehabilitation tool, we first needed to develop and evaluate the tactile devices to ensure that realistic sensations are generated; this is the focus of this paper.

Many tactile devices have been reported in the literature but, despite considerable advancements, there is a distinct paucity of studies evaluating the perceptual experience of their use. This research was undertaken by a multidisciplinary team, with a collaborative, user-led approach to development. The team consisted of engineers, psychologists and clinicians who equally contributed opinions to designing the tactile devices.

Three tactile devices were developed (e.g. Fig. 1), capable of giving rise to realistic sensations of static interaction with virtual objects. The devices were qualitatively evaluated with human participants and iteratively redeveloped based on the feedback. Seven non-impaired participants took part (4 male, 3 female; age 23-59yrs, mean=36.4, $\mathrm{SD}=13.5$ ). Non-impaired participants ensured we did not give people with impaired sensation noxious stimuli, and established a normative baseline before evaluating on people with stroke. Ethical approval was granted by the Faculty of Health Science's Ethics Committee, University of Southampton (ref 2010-025).

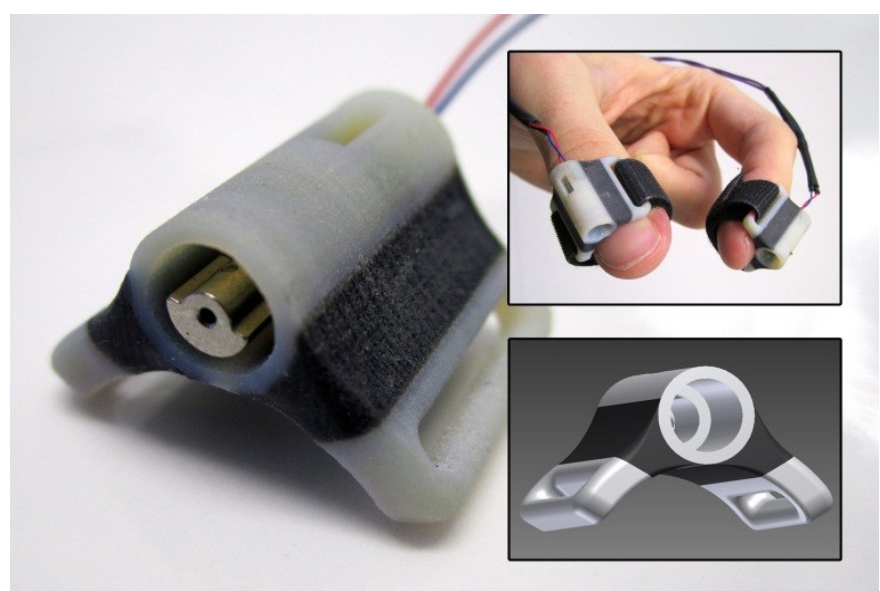

Figure 1: 'Vibration' tactile device (inset-top: devices mounted on the thumb and index finger; inset-bottom: CAD drawing of the casing).

This paper is structured as follows: Section 2 gives an overview of the existing research on tactile devices, followed by details of the vibration (Section 3), motor-driven 'squeezer' (Section 4), and SMA (Section 5) devices that have been developed in this research. For clarity, the results 
of the human evaluation are included at the end of each section. In particular, Section 5 illustrates the iterative design and evaluation process adopted, showing three cycles of userinformed redesign. Section 6 discusses the results, draws conclusions and identifies areas for further investigation.

\section{Tactile Devices}

The field of tactile devices has received significant research interest over the past few decades [8]. Devices proposed in the literature include those using piezoelectrics [9], electrocutaneous stimulation [10], electrorheological fluids [11] and electroactive polymers [12]. In this research, we consider three different technologies: vibration, a motordriven 'squeezer' and Shape Memory Alloys (SMAs).

Vibration, or vibro-tactile stimulation, is the most widely used tactile interface, with numerous commercial applications [13] including mobile phones [14] and game controllers [15]. The CyberTouch system [7], a commercially available general purpose 'haptic glove', is marketed for many virtual reality applications, including computer aided design and robotic surgery. The CyberTouch glove contains a vibro-tactile stimulator on the dorsum (top) of each finger and the palm. Each stimulator can be individually addressed to vary the vibration $(0-125 \mathrm{~Hz}$, with a maximum peak-to-peak force of $1.2 \mathrm{~N}$ ), and can deliver pulses or sustained stimulation.

Vibro-tactile stimulation has been utilised in a number of research studies, including the Mobile Music Touch system [16], which used a glove with vibration motors attached to each of the fingers to both assist piano playing and in rehabilitating patients with spinal cord injury. Israr et al. reported a system using twelve vibro-tactile stimulators (each vibrating at $180-200 \mathrm{~Hz}$ ) placed on a patient's body, to control breathing patterns during radiation therapy [17]. However, in both of these examples (and in a large number of vibro-tactile applications), vibration is used as an indicator rather than a realistic sensation of touch; that is, the user does not think that they are actually touching an object, but the stimulation acts as a cue that they can act upon. The mechanoreceptors in the fingertip that detect vibration have a large receptive field (the area over which they detect stimuli), and hence the source of vibration cannot be accurately localised; it is commonly accepted that one stimulator per fingertip is sufficient [18]. In addition to tactile stimulation, vibration also provides kinaesthetic stimulation through the 'shaking' of the finger.

In order to provide a more realistic sensation of touching a surface, researchers experimented with tightening a belt around the fingertip, thus applying pressure. The device reported by Minamizawa et al. [19] consists of two ø10mm motors mounted on the dorsum of the fingertip. By turning both motors in different directions, a belt is pulled up against the fingertip, giving an impression of pressure. By turning both motors in the same direction, the belt moves from sideto-side over the fingertip, giving an impression of 'shear' force. The devices are attached to the finger by means of a Velcro band around the second phalanx; this is necessary as the band cannot be secured around the first phalanx due to the presence of the belt. This attachment mechanism results in restricted bending of the finger, as it is effectively 'splinted'. This issue was partially addressed via the integration into a glove (whereby the devices were attached to the glove rather than the finger), providing stimulation to all four fingers, the thumb and the palm [20]. However issues of spasticity will affect the ability of a person recovering from stroke to wear such a glove, as donning and doffing becomes difficult. The devices are also reasonably large and heavy, restricting the movement of the fingers. This is a particular concern for its use with stroke patients, who already have restricted mobility. Some of these issues were rectified in a device reported by Aoki et al. [21], which was lightweight $(1.4 \mathrm{~g})$ and capable of delivering a force of $420 \mathrm{mN}$. The authors found that tightening a single wire $(\varnothing 3 \mathrm{~mm})$ as opposed to a band made it easier to perceive. However, it is unlikely that this mimics the sensation of touching a planar surface.

Other materials can also offer the ability to apply pressure to the fingertip. Shape Memory Alloys (SMAs), such as NiTi (Nickel-Titanium, commonly referred to as Nitinol), are functional materials which can remember a shape (trained by applying a high temperature). When at a low (room) temperature, the alloy is in Martensite phase and behaves like a deformable metal. When heated to its transformation temperature (for example, by application of an electric current), the SMA changes to Austenite where it 'remembers' its pre-trained shape. Furthermore, in the transformation to Austenite, the length of the SMA wire reduces by 4-5\% [22].

SMA wires have been proposed for a number of different tactile devices, including their use as active helical springs (where heating causes it to contract, driving pins against the fingertip) [23] and linear actuators (where the shortening of the SMA wire directly retracts a sprung pin) [22]. A commonly encountered problem with SMA wires is that they take a significant period of time to return to Martensite (through cooling); hence, both of these reported designs use external forces, applied by springs or permanent magnets, to combat this. The concept investigated in this research is based upon a design by Scheibe et al. [24], where thimbles worn on the fingertips contain $\varnothing 80 \mu \mathrm{m}$ SMA wires 'wrapped' around the finger. Upon the application of an electric current, the SMA wires shorten by $1.5-2.5 \mathrm{~mm}$, applying pressure to the fingertip. Heating of the SMA wires is performed by using a Pulse Width Modulated (PWM) signal, with a high duty cycle applied for the first $40 \mathrm{~ms}$ (during transformation from Martensite to Austenite), followed by a lower duty cycle for the remainder of the stimulation period to provide a 'pulsing' sensation. As the heated wire is in direct contact with the wearer's skin, care has to be taken to ensure that the wires do not burn the skin or cause discomfort.

The following three sections discuss the design, development and evaluation of the devices investigated in this project.

\section{3 'Vibration' Tactile Device}

To generate a vibration in a small and lightweight device, we opted to use a miniature vibration motor (a motor with an 
offset mass, as found in most mobile phones and pagers). These are commonly offered in two different formats, 'cointype' (a cylindrical disc, as used in the Mobile Music Touch system [16]) and 'bar-type' (a cylindrical tube). In both formats, the offset mass rotates around the axis of the cylinder. Therefore, we deemed the coin-type motor to be unsuitable as, when attached to the finger, the generated forces would act side-to-side rather than the normal force desired to create a sensation of pressure. The chosen $ø 4 \mathrm{~mm}$ motor (Precision Microdrives, 304-002) had a nominal speed of $12000 \mathrm{rpm}(200 \mathrm{~Hz})$, giving a nominal vibration of $6 \mathrm{~ms}^{-2}$. The device was driven using PWM, allowing frequency (7$100 \mathrm{~Hz})$ and duty cycle $(20-80 \%)$ adjustment.

A number of different designs were considered for mounting the device on the fingertips. Initial versions were based around a 'thimble' worn on the fingertip, but were disregarded as they were deemed too large and heavy, and as they caused significant vibration damping. Casings were created using a $3 \mathrm{~d}$ printer (Objet Connex), allowing rapid prototyping of the designs under consideration (essential due to the iterative nature of this research). The final device is shown in Fig. 1. The motor chamber is moulded to securely hold the motor in place while also providing space for the offset mass to rotate. Small holes were left in the rear of the chamber for wiring, and a ventilation hole included on the top. The device is attached to the finger via Velcro straps which are secured through eyelets on either side of the case. Softer 'rubber-like' sections (black areas in Fig. 1) were included to allow identical devices to be comfortably attached to the thumbs and fingers of different people. While these softer sections damped the vibration (and hence its translation to the opposite side of the finger), this isolation and stability proved to be advantageous and reduced wear on the eyelets.

The design allows for flexible mounting of the device, which can be worn on the dorsum or ventrum (underside) of any phalanx on the finger or thumb. To increase perception in the pulp of the fingertip, a textured disc was inserted between the strap and finger, and was also tested.

\subsection{Results: 'Vibration' Tactile Device}

The general consensus from user feedback on the vibration device was that it provided a good 'indication' of touch, but not a realistic sensation; commenting that it felt more like touching a vibrating object, rather than holding something in the hand. Feedback was also received commenting that the devices felt unnatural and annoying. This perception remained unchanged whether the device was located on the dorsum or ventrum of the fingertip, contributable to the large receptive field of the mechanoreceptors sensitive to vibration. The inclusion of the textured discs was found to have little additional benefit, especially considering the resulting increase in size, weight and attachment complexity.

\section{4 'Motor-Driven Squeezer' Tactile Device}

The 'motor-driven squeezer' device (referred hereon as the 'squeezer') is based on the design reported by Minamizawa et al. [19], discussed in Section 2. Our device (Fig. 2) consists of two miniature motors (Maxon Motor, RE6 4.5V motors, with a $15: 1$ gearhead) used to pull a flexible band to apply compression to the pulp of the fingertip. To reduce the device's size and weight, these $ø 6 \mathrm{~mm}$ motors were chosen over the $ø 10 \mathrm{~mm}$ variants used by Minamizawa et al. The belt (replaceable in order to investigate different fabrics and materials) was attached to the motors via plastic extensions clamped onto the spindles. Two case sizes were created, one for use on fingers, and one (slightly wider) for use on thumbs. It was found that these two sizes fitted all of the participants comfortably.

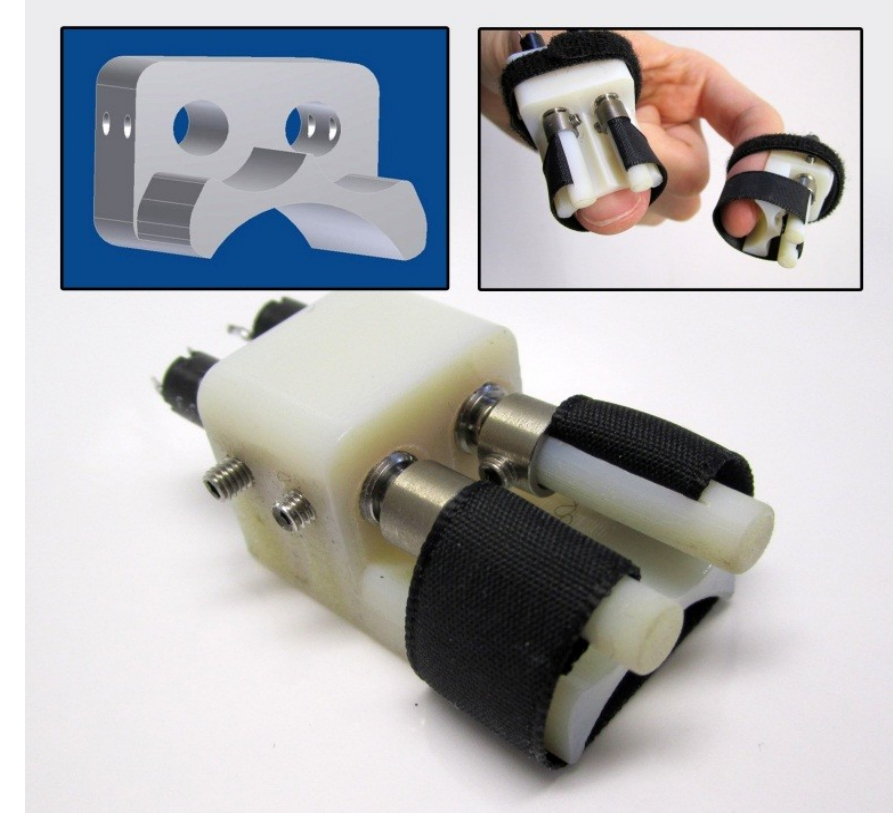

Figure 2: 'Motor-driven squeezer' tactile device (inset-left: CAD drawing of the casing; inset-right: devices mounted on the thumb and index finger).

The motors are driven using PWM. This signal is generated from an MSP430 microcontroller, and can drive four motors (i.e. two devices) concurrently, allowing independent control over duty cycle $(0-100 \%)$ and frequency $(50 \mathrm{~Hz}-5 \mathrm{kHz})$. The operation of the MSP430 is controlled by either a computer via RS232 (allowing parameters such as duty cycle and frequency to be entered) or via switches to start and stop stimulation (permitting operation without a computer).

Two control options were considered for driving the motors. The first, position sensing, would have been preferable (due to its ability to identify the precise rotation of the motor) but was not feasible as miniature COTS sensors were not available for the selected motors. The second option, and adopted in this design, was to monitor the current through the motor and hence the torque it delivers. The current is sampled from a small (1 $\Omega$ ) shunt resistor through a difference amplifier, by an ADC on the MSP430. This is subsequently used by the PID control scheme. Through this mechanism, the software allows different torques, and hence constriction speeds, to be delivered by the motors. A benefit of current sensing is that the sensing electronics does not need to be on the device (and hence finger), but instead can be located with the control circuitry (for example on the arm, waist or table). 


\subsection{Results: 'Motor Driven Squeezer' Tactile Device}

The initial objective of the squeezer device was to simulate a flat pressure at the ventrum of the fingertip. Participants generally reported a feeling of instantaneous pressure or touching an object quickly, rather than picking up an object. This device was also described as cumbersome and not suitable for its intended purpose. Some participants also reported a sensation of constriction around the finger rather than pressure on the pulp. As anticipated, the attachment (Velcro straps on the second phalanx) produced an effect analogous to splinting the end joint of the finger, thus reducing users' mobility.

\section{5 'Shape Memory Alloy' Tactile Device}

As highlighted in Section 2, SMAs have found application in tactile devices through a range of different methods. To provide constriction of the fingertip (similar to the 'squeezer' device reported in Section 4), we chose to use their ability to shorten in length when heated. The development of this device illustrates the iterative design and evaluation process adopted in this project. This cycle was repeated three times, with participant-informed improvements made at each step; details of these are discussed in the following subsections.

\subsection{Mk1: Initial Design}

Initial designs for the SMA device consisted of two moulded plates mounted on the dorsum and ventrum of the fingertip, which were pulled together by SMA wires when an electric current was applied. However, through preliminary evaluation, it was reported that the device was large, restricted movement, and provided a weak sensation. Instead, it was found that direct contact of the SMA wires with the finger created a more noticeable sensation, hence we adopted and developed the design reported by Scheibe et al. [24].

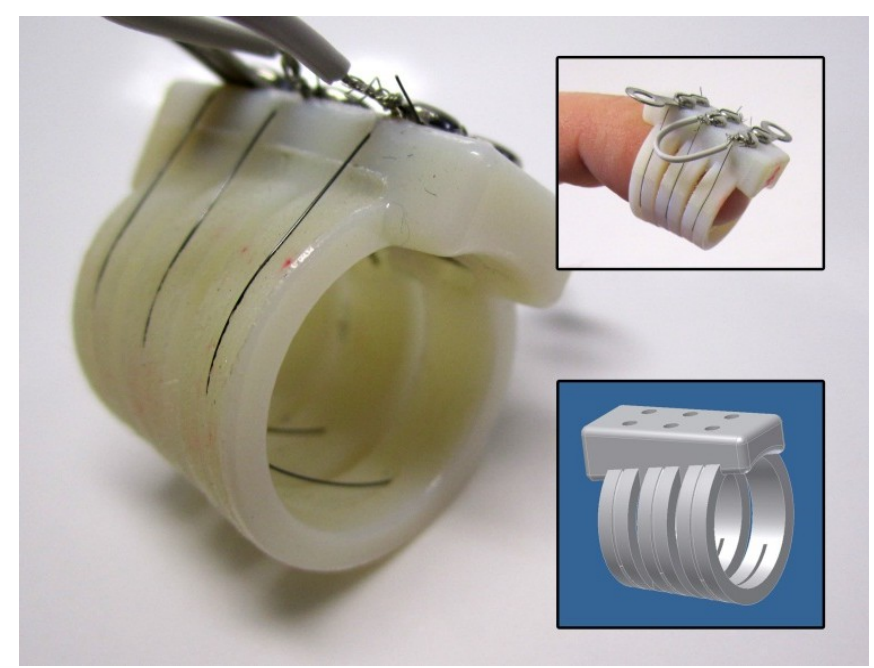

Figure 3: Mk1 of the 'SMA' tactile device (inset-top: device mounted on the index finger; inset-bottom: CAD drawing of the casing).

The first iteration (Fig. 3) was largely unchanged from the reported device. A trade-off between the rate-of-heating (i.e. the speed at which stimulation is applied) and the temperature that the wires (in contact with the skin) reach, proved a major issue. The three $\varnothing 200 \mu \mathrm{m}$ SMA wires (spaced $6 \mathrm{~mm}$ apart) were driven by a PWM signal, allowing the stimulation to be varied by adjusting the duty cycle and frequency. Nine versions of the device were created in order to accommodate different finger sizes. To ensure that each device gave a comparable level of stimulation, the voltage for each was adjusted accordingly. This was necessary as the various casings (used simultaneously on the forefinger and thumb) used different length SMA wires, and hence resistances.

\subsubsection{Mk1 Results}

Human evaluation of the Mk1 device reported that it felt like slowly touching a hot 'spongy' object and that, rather than a surface, the three wires were individually perceivable. This is likely to be directly attributable to the distance between the wires being greater than the $2 \mathrm{~mm}$ two-point discrimination limit that can be detected at the fingertip [25].

\subsection{Mk2: Reduced Wire-Spacing}

The second iteration $(\mathrm{Mk} 2)$ of the device addressed the issue raised concerning the perception of individual wires. This was achieved by reducing the wire spacing to $1.5 \mathrm{~mm}$ (below the two-point discrimination threshold [25]), while maintaining a large contact area through 12 SMA wires. Due to the reduced wire spacing, attaching them to the casing via screw clamps was unfeasible. For this reason, the wires were clamped between two metal bars, which can be seen in Fig. 4.

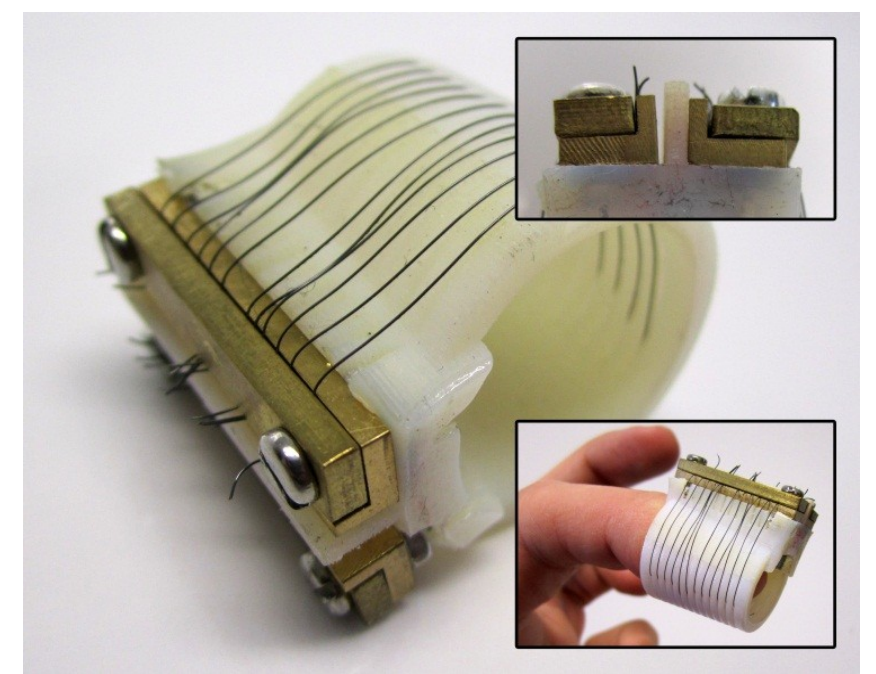

Figure 4: Mk2 of the 'SMA' tactile device (inset-top: close-up of the wire clamp; inset-bottom: device mounted on the index finger).

\subsubsection{Mk2 Results}

Evaluation of this device found that individual wires were not perceived; rather it felt like touching a hot surface. However, it was also reported that the level of stimulation was considerably lower than the Mk1 device (in some cases it was unnoticeable), and that the wires got hot very quickly (within a second). It was felt that the device was larger, heavier and more restrictive than the Mk1 device. Technical issues were also experienced with the 'clamp' failing and releasing wires, and all 12 wires rarely made contact with the finger. 


\subsection{Mk3: Improved Control}

The third iteration $(\mathrm{Mk} 3)$ of the device addressed the issues that were raised regarding reduced stimulation, increased temperature, and wearability. Issues with wire attachment were resolved through the use of a single SMA wire coiled (7 turns) around the device, clamped and coated in silver epoxy (shown in Fig. 5). This also enabled the size and weight of the device to be reduced, thus increasing wearability.

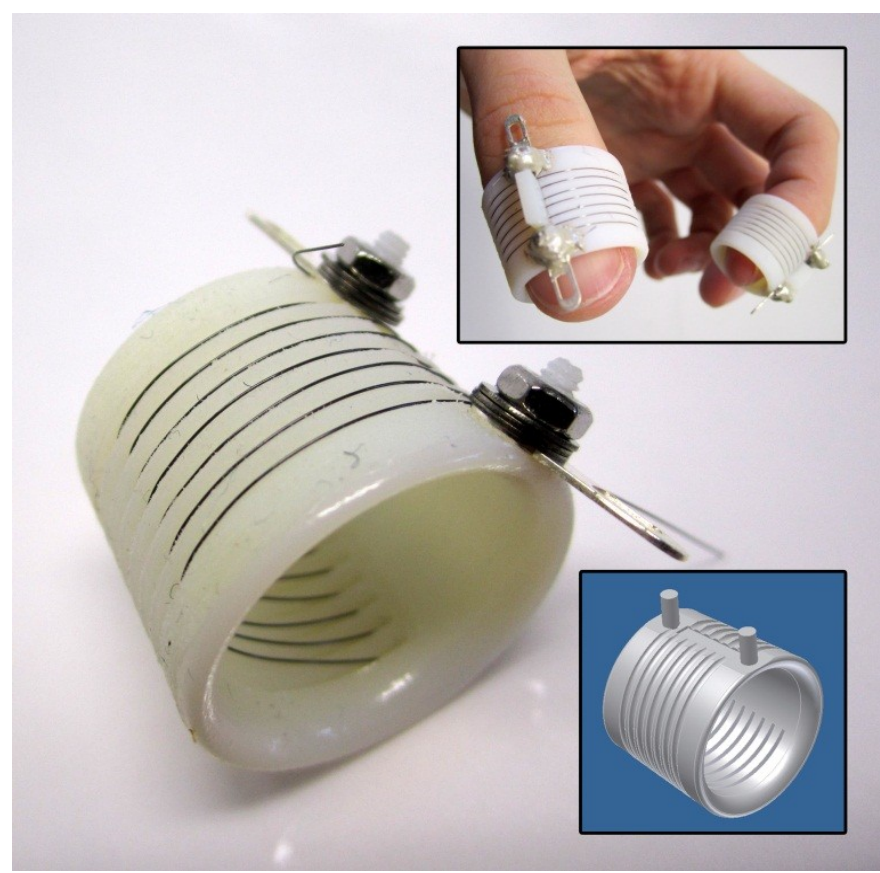

Figure 5: Mk3 of the 'SMA' tactile device (inset-top: devices mounted on the thumb and index finger; inset-bottom: CAD drawing of the casing).

To address the issues concerning weak, slow and high temperature stimulation, an improved control scheme was adopted. With the previous devices, a constant PWM signal was used to drive the SMA wires; this means that to keep the temperature within comfortable limits during the stimulation period, the application of pressure is gradual (hence feeling like touching a 'spongy' object). This control scheme is depicted graphically by the dashed line in Fig 6.

Instead of using the 'two-state' duty-cycle scheme reported by Scheibe et al. [24] (which would require manual calibration for different size devices, and introduce considerable variability in the level of applied stimulation), PID resistance-control was implemented on the MSP430 microcontroller [22] [26]. This operates on the principle that as the phase of the SMA changes between Austenite and Martensite, its electrical resistance also changes. Therefore, by sensing the resistance of the wire, its length can be identified and hence controlled. The controller operated by applying a PWM signal of a preset duty-cycle (which defines the rate at which stimulation is applied) until the resistance of the SMA $\left(R_{S M A}\right)$ decreased below a threshold $\left(R_{\text {threshold }}\right)$. Once this threshold is reached, the PID controller maintains a preset resistance $\left(R_{\text {steady }}\right)$ by adjusting the duty cycle (defined as a percentage of the room-temperature resistance, and allowing control over the level of stimulation applied). This scheme, depicted graphically by the solid line in Fig. 6, allows independent variation of these parameters while preventing the wires from reaching uncomfortable temperatures. In addition, the casing was redesigned using a tapered approach (see Fig. 5)
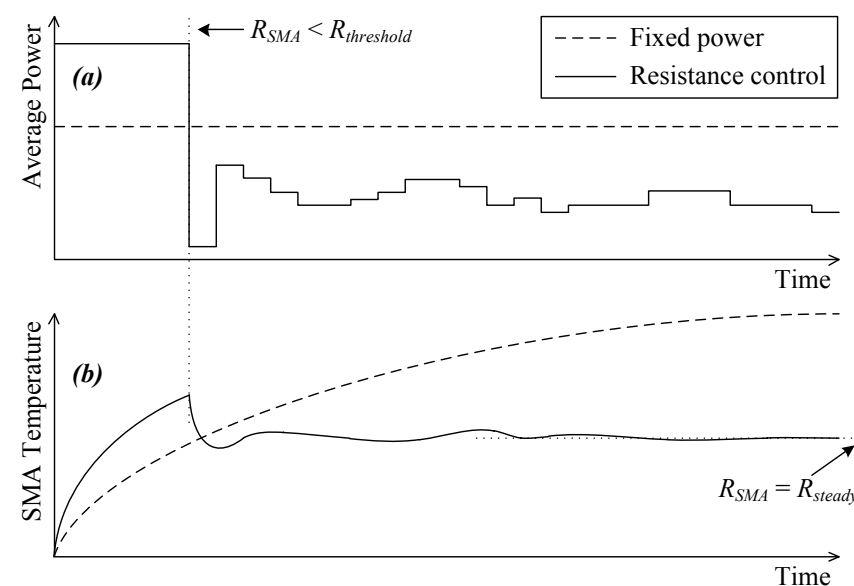

Figure 6: Illustration of the effect of different control schemes for heating the SMA wires, a) the duty cycle, or average power, put through the SMA wires, and b) the temperature of the SMA wire.

\subsubsection{Mk3 Results}

User feedback from the Mk3 device reported that the improved tapered design of the case helped increase the surface contact area between the wires and the fingertip along the length of the case. This alteration therefore optimised the stimulation received by each participant and helped with the potential wearability issues reported in previous iterations. The temperature concerns reported by the participants at the Mk2 stage were not alleviated in Mk3. Although improvements were reported, participants described being aware of a warmth generated from the Mk3. However, participants reported feeling sensations of gentle pressure or pulsations, and sensations similar to touching a firm object.

\section{Discussion and Conclusions}

The previous sections have evaluated and reported on a userled, iterative design process for three tactile devices; vibration, the motor squeezer, and the three iterations of the SMA device. The SMA devices showed that perceivable vibration and/or heat distracted from the perception of touching an object; generally sensations were described as handling malleable objects, such as a plastic cup. Increasing the duty cycle and frequency of stimulation provided the sensation of handling a more solid object. Comparisons between the devices showed the SMA to be more convincing in delivering sensations akin to grasping objects (static exploration), whilst the vibration device provided information about object surface properties (dynamic exploration). The squeezer device was found to be more cumbersome than the other devices, and reduced mobility through splinting. We are currently investigating the use of vibration as an instantaneous force (replicating dynamic impact forces rather than static pressure), especially in conjunction with static pressure (for example using the squeezer or SMA devices). 
This project has highlighted the importance of multidisciplinary and truly collaborative methodological approaches to device development. Adopting an approach of involving users in the design process, from the initial stages, has proven to be very insightful in the development of these devices. Commercially accepted solutions to tactile stimulation have proven to be illustrative, but not provide realistic sensation. The devices presented in this paper highlight potential solutions, and have been designed and developed with user involvement, therefore having the potential to be acceptable to a range of applications including rehabilitation, gaming and immersive virtual environments. We are now evaluating the developed devices with impaired participants, and will report on our findings in the future.

\section{Acknowledgements}

This research was funded by an 'Adventures in Research' grant from the University of Southampton's Faculties of 'Engineering, Science and Mathematics,' and 'Medicine, Health and Life Sciences.'

\section{References}

[1] M. McLaughlin, A. Rizzo, Y. Jung, et al., "HapticsEnhanced Virtual Environments for Stroke Rehabilitation," in Proc. ISPI, Cambridge, MA, 2005.

[2] K. Pearson, "Motor systems," Current Opinion in Neurobiology, 10, pp. 649-654, (2000).

[3] M. Yekutiel, Sensory Re-Education of the Hand After Stroke. London, UK: Whurr Publishers, 2000.

[4] A. Rizzo, M. Mclaughlin, Y. Jung, et al., "Virtual Therapeutic Environments with Haptics: An Interdisciplinary Approach for Developing Post-Stroke Rehabilitation Systems," in Proc. Computers for People with Special Needs, 2005, pp. 70-76.

[5] S. Subramanian, L. A. Knaut, C. Beaudoin, et al., "Virtual Reality Environments for Post-Stroke Arm Rehabilitation," J. Neuroengineering and Rehabilitation, 4, (2007).

[6] R. S. Dahiya, G. Metta, M. Valle, et al., "Tactile Sensing: From Humans to Humanoids," IEEE Trans. Robotics, 26, pp. 1-20.

[7] CyberGlove Systems LLC. [Online]. Available: www.cyberglovesystems.com/. [Accessed: Jan, 2011].

[8] V. G. Chouvardas, A. N. Miliou, and M. K. Hatalis, "Tactile Displays: Overview and Recent Advances," Displays, 29, pp. 185-194, (2008).

[9] V. Levesque, J. Pasquero, and V. Hayward, "Braille Display by Lateral Skin Deformation with the STReSS2 Tactile Transducer," in Proc. World Haptics, 2007, pp. 115-120.

[10] H. Kajimoto, "Electro-tactile Display with Real-Time Impedance Feedback," in Proc. EuroHaptics, Amsterdam, Netherlands, 2010, pp. 285-291.

[11] P. M. Taylor, D. M. Pollet, A. Hosseini-Sianaki, et al., "Advances in an electrorheological fluid based tactile array," Displays, 18, pp. 135-141, (1998).
[12] K. Ig Mo, J. Kwangmok, K. Ja Choon, et al., "Development of Soft-Actuator-Based Wearable Tactile Display," IEEE Trans. Robotics, 24, pp. 549-558, (2008).

[13] A. U. Alahakone and S. M. N. A. Senanayake, "Vibrotactile feedback systems: Current trends in rehabilitation, sports and information display," in Proc. IEEE Int'l Conf. Advanced Intelligent Mechatronics, 2009, pp. 1148-1153.

[14] S. Brewster, F. Chohan, and L. Brown, "Tactile feedback for mobile interactions," in Proc. Conf. Human factors in Computing Ssystems, San Jose, CA, 2007.

[15] D. Chang, "Haptics: gaming's new sensation," Computer, 35, pp. 84-86, (2002).

[16] T. Markow, N. Ramakrishnan, K. Huang, et al., "Mobile Music Touch: Vibration stimulus in hand rehabilitation," in Proc. Pervasive Computing Technologies for Healthcare (PervasiveHealth), pp. 1-8.

[17] A. Israr, D. P. Eng, S. S. Vedam, et al., "A low cost vibrotactile array to manage respiratory motion," in Proc. World Haptics, 2009, pp. 619-620.

[18] A. Fisch, C. Mavroidis, J. Melli-Huber, et al., "Haptic Devices for Virtual Reality, Telepresence, and HumanAssistive Robotics," in Biologically Inspired Intelligent Robots, Y. Bar-Cohen and C. L. Breazeal, Eds. Washington: SPIE, 2003, pp. 73-102.

[19] K. Minamizawa, S. Fukamachi, H. Kajimoto, et al., "Gravity grabber: wearable haptic display to present virtual mass sensation," in Proc. SIGGRAPH Emerging Technologies, San Diego, California, 2007.

[20] K. Minamizawa, S. Kamuro, S. Fukamachi, et al., "GhostGlove: Haptic Existence of the Virtual World," in Proc. SIGGRAPH New Tech Demos, Los Angeles, CA, 2008.

[21] T. Aoki, H. Mitake, D. Keoki, et al., "Wearable haptic device to present contact sensation based on cutaneous sensation using thin wire," in Proc. Int'l Conf. Advances in Computer Enterntainment Technology, Athens, Greece, 2009.

[22] P. M. Taylor, A. Moser, and A. Creed, "A sixty-four element tactile display using shape memory alloy wires," Displays, 18, pp. 163-168, (1998).

[23] T. Matsunaga, K. Totsu, M. Esashi, et al., "Tactile display for 2-D and 3-D shape expression using SMA micro actuators," in Proc. Microtechnology in Medicine and Biology, 2005, pp. 88-91.

[24] R. Scheibe, M. Moehring, and B. Froehlich, "Tactile Feedback at the Finger Tips for Improved Direct Interaction in Immersive Environments," in Proc. IEEE Symposium 3D User Interfaces, 2007.

[25] J. Dargahi and S. Najarian, "Human tactile perception as a standard for artificial tactile sensing-a review," $J$. Medical Robotics and Computer Assisted Surgery, 1, pp. 23-35, (2004).

[26] J. Urata, T. Yoshikai, I. Mizuuchi, et al., "Design of high D.O.F. mobile micro robot using electrical resistance control of shape memory alloy," in Proc. IEEE Int'l Conf. Intelligent Robots and Systems, 2007, pp. 3828-3833. 